\title{
INTERAÇÃO DE ÁTOMOS LEVES COM CLUSTERS DE METAIS DE TRANSIÇÃO
}

Eduardo Pires Cassús*

CENPES - Petrobras, Av. Horácio de Macedo, 950, 21941-915 Rio de Janeiro - RJ, Brasil

Sérgio de Paula Machado, Francisco M. S. Garrido, Marta E. Medeiros e Juan Omar Machuca-Herrera

Departamento de Química Inorgânica, Instituto de Química, Universidade Federal do Rio de Janeiro, CP 68563, 21945-970 Rio

de Janeiro - RJ, Brasil

Recebido em 1/11/10; aceito em 28/4/11; publicado na web em 14/6/11

INTERACTION OF SMALL ATOMS WITH TRANSITION METAL CLUSTERS. Density Functional Theory (DFT) calculations on the interactions of small atoms $(\mathrm{H}, \mathrm{C}, \mathrm{O}$, and $\mathrm{S})$ on first-row transition metal clusters were performed. The results show that the adsorption site may vary between the metal surface and the edge of the cluster. The adsorption energies, adatom-nearest neighbor and adatom-metal plane distances were also determined. Finally, the authors present a discussion about the performance of these metals as anodes on solid oxide fuel cells. The results obtained agree with empirical data, indicating that the theoretical model used is adequate.

Keywords: density functional theory; solid oxide fuel cells; transition metal.

\section{INTRODUÇÃO}

A posição dos átomos adsorvidos em uma superfície metálica é uma característica importante na descrição dos processos que dependem da adsorção. Além disso, a interação de átomos com superfícies metálicas tem um papel preponderante em vários processos catalíticos e eletroquímicos. Por isso, espera-se que tais parâmetros fundamentais sejam rigorosamente descritos em termos de energia. O nível atual de desenvolvimento da Química Computacional permite uma abordagem mais próxima do real nestes tópicos. ${ }^{1,2}$ Os sítios de adsorção em superfícies metálicas diferem principalmente no número de primeiros vizinhos (número de coordenação) e simetria bidimensional. Como a interação adsorvente-adsorbato é atrativa, espera-se que um átomo adsorvido interaja com o maior número de átomos metálicos, a fim de reduzir a energia de interação.

Cálculos usando a teoria do funcional de densidade (DFT) foram usados a fim de se estimar a energia de adsorção de pequenos átomos sobre superfícies metálicas. Espera-se que tais cálculos complementem o conhecimento adquirido com dados experimentais. Diversas reações de interesse industrial, bem como alguns processos de conversão de energia, como o desenvolvimento de células a combustível, podem se beneficiar destes esforços combinados. Neste assunto específico, o papel de átomos como $\mathrm{H}, \mathrm{C}, \mathrm{O}$ e S é importante, pois estas espécies estão presentes em combustíveis (seja na forma de constituintes ou de contaminantes) usados em células a combustível. ${ }^{3-5}$

Ao se considerar o mecanismo mais apropriado para descrever os fenômenos que ocorrem em anodos do tipo cermet, é preciso considerar diferentes rotas reacionais que possam ocorrer nas superfícies anódicas, formadas pelas interfaces entre metais, eletrólitos e gás combustível (ponto triplo, TPB). O transporte de gás e íons ocorre em várias etapas: difusão das espécies reativas pela fase gasosa; adsorção dissociativa de gases na superfície do metal ou eletrólito (formação de $\mathrm{H}_{\mathrm{ad}}$ e $\mathrm{O}_{\mathrm{ad}}$ ); difusão das espécies adsorvidas na superfície e, transferência de carga na superfície metálica, seguindo a equação geral: ${ }^{6}$

*e-mail: cassus@petrobras.com.br

$$
\mathrm{O}_{\mathrm{O} \text { (eletrólito) }}^{\mathrm{x}}+[]_{\text {metal }}=\mathrm{O}_{\text {metal }}+\mathrm{V}_{\mathrm{O}^{\cdots(\text { (eletrólito) }}}+2 \mathrm{e}_{\text {(eletrodo) }}^{-}
$$

onde, [ $]_{\text {metal }}$ refere-se a um sítio ativo na superfície metálica e os demais símbolos seguem a notação de Kroger-Vink.

De acordo com alguns resultados empíricos, uma das etapas lentas do processo de oxidação de combustível está associada à transferência de íons oxigênio do eletrólito ao metal (oxygen spillover) ${ }^{6-10}$ Outros grupos sugerem que a oxidação do hidrogênio em anodos de níquel é controlada por um processo de dois eletrodos na superfície das partículas de níquel e um processo de transferência de carga na superfície do eletrodo como mostrado nas Equações 2 e 3:11,12

$$
\mathrm{H}_{\text {Metal }}=\mathrm{H}_{\text {ad(eletrólito, próximo ao TPB) }}+[]_{\text {metal }}
$$

$\mathrm{O}_{\mathrm{O}}^{\mathrm{x} \text { (eleterólito) }}+\mathrm{H}_{\text {ad(eletrólito, próximo ao TPB) }}=\mathrm{H}_{2} \mathrm{O}_{\text {(gás) }}+2 \mathrm{e}_{\text {(eletrólito, próximo ao TPB) }}^{-}+$ $\mathrm{V}_{\mathrm{o}}$ ( eletrólito)

A Equação 3 representa a reação de transferência de carga na superfície do eletrodo, próximo ao ponto triplo, sendo a etapa lenta do processo.

Outros grupos, como os de Ihara e Biieberle et al., apresentaram modelos alternativos para os cermets Ni/YSZ, nos quais todas as etapas ocorrem sobre a superfície do níquel. ${ }^{13,14}$ Pelo mecanismo proposto pelo grupo de Ihara, todas as espécies neutras $\mathrm{H}, \mathrm{O}$ e $\mathrm{H}_{2} \mathrm{O}$ estariam em equilíbrio na superfície metálica, e as etapas seriam: ${ }^{9,13-15}$

etapa 1

$$
\mathrm{H}_{2 \text { (gás) }}+2[]_{\mathrm{Ni}}=2 \mathrm{H}_{\mathrm{Ni}}
$$

etapa 2

$$
\mathrm{O}_{\mathrm{O}}^{\mathrm{x}} \text { (eletr }
$$$$
\text { [1 }=\mathrm{O}^{\mathrm{N}}+\mathrm{V}
$$$$
+2
$$

etapa 3

$$
2 \mathrm{H}_{\mathrm{Ni}}+\mathrm{O}_{\mathrm{Ni}}=\mathrm{H}_{2} \mathrm{O}_{\mathrm{Ni}}+2[]_{\mathrm{Ni}}
$$

etapa 4

$$
\mathrm{H}_{2} \mathrm{O}_{\mathrm{Ni}}=\mathrm{H}_{2} \mathrm{O}_{\text {(gá) }}+[]_{\mathrm{Ni}}
$$

O terceiro passo é a etapa lenta do processo. Por isso, de acordo com este modelo, é importante compreender as energias envolvidas nos processos de adsorção de átomos de $\mathrm{H}$ e $\mathrm{O}$ sobre superfícies me- 
tálicas, pois elas são fundamentais para a cinética do processo e, por isso, explicariam os resultados experimentais obtidos por Setoguchi et al. em relação à eficiência de células de óxido sólido. ${ }^{16}$

É conhecido o fato de que o níquel é um catalisador excelente não só para a reforma a vapor, mas também para o craqueamento do hidrogênio, de modo que depósitos de carbono em sua superfície ocorrem rapidamente quando um hidrocarboneto é usado como combustível, a menos que um excesso de vapor seja empregado para garantir a reforma completa, com uma perda de eficiência. ${ }^{6,17-20} \mathrm{O}$ mesmo problema ocorre com ferro e cobalto, mas não é observado com o cobre, que é menos eficiente. Outra questão envolvendo o cobre é a preparação do anodo que, devido a sua instabilidade sob altas temperaturas, sofre sinterização. Por esses motivos, o desenvolvimento de eletrodos adequados para hidrocarbonetos ainda é uma questão a ser solucionada. ${ }^{18}$

Outro aspecto importante a ser considerado é o envenenamento do metal por enxofre. Este processo pode ocorrer pela adsorção do enxofre atômico ou pela formação de sulfetos metálicos, como observado pela espectroscopia Raman em amostras de níquel. ${ }^{21-24}$ A determinação do mecanismo para a adsorção do enxofre é muito importante para a fabricação de novos anodos mais tolerantes a este tipo de contaminação.

As energias de adsorção para vários átomos sobre diferentes superfícies metálicas são de fundamental importância. Estes dados não estão disponíveis para todos os elementos da primeira série de transição. Existem trabalhos teóricos sobre o tema, mas não cobrem todos os elementos, o que deixa o campo ainda aberto..$^{25-28}$

Neste trabalho, são apresentados resultados usando a abordagem da teoria do funcional de densidade a fim de se estudar as geometrias de equilíbrio e energias de adsorção de átomos leves (H, C, O e S) sobre clusters de elementos da primeira série de transição (Fe, Co, $\mathrm{Ni}, \mathrm{Cu}$ e Zn). Os clusters modelo possuem sete átomos, respeitando as geometrias encontradas em bancos de dados cristalográficos. Ao final do trabalho, é feita uma comparação com pesquisas anteriores a fim de validar a abordagem empregada. Verificou-se também a validade dos resultados qualitativos em relação aos mecanismos propostos por outros autores, para a oxidação do hidrogênio sobre superfícies metálicas.

\section{METODOLOGIA COMPUTACIONAL}

No presente trabalho, clusters com sete átomos de metal foram criados para simular a adsorção de átomos em superfícies metálicas. A fim de se manter a consistência do modelo, as coordenadas usadas dos átomos de metal foram obtidas a partir de bases de dados cristalográficas. ${ }^{29}$ Além disso, as coordenadas dos átomos metálicos foram fixadas e apenas as coordenadas dos átomos adsorvidos foram otimizadas, seguindo-se a metodologia usada por outros pesquisadores. A abordagem empregada apresenta boa concordância com os experimentos e não requer um grande esforço computacional..$^{25}$

A interação dos átomos leves $(\mathrm{H}, \mathrm{C}, \mathrm{O}$ e $\mathrm{S})$ com diferentes clusters metálicos foi estudada por cálculos de primeiros princípios via teoria do funcional de densidade (DFT) usando o funcional de troca-correlação B3LYP e o funcional "puro" XPBE96, como implementados no pacote NWchem. ${ }^{30-32}$ A escolha do primeiro funcional se baseia em seu grande uso na atualidade e a do segundo para comparação, pois este não superestima as ligações covalentes. Para os átomos metálicos, usaram-se potenciais de caroço efetivo na descrição dos orbitais internos e as camadas externas foram descritas explicitamente usando-se a base LANL2DZ. Para os átomos leves (ou seja, H, C, O e S), a base 6-31G foi empregada. A base 6-31G é uma base de valência dividida que apresenta boa relação entre o custo computacional e a qualidade dos resultados para elementos leves. Já a descrição eletrônica dos metais de transição torna-se mais conveniente se for realizada com o auxílio de uma base com potencial de caroço efetivo. As vantagens dessa abordagem envolvem a economia de esforço computacional no que diz respeito aos elétrons mais internos, priorizando-se o cálculo da energia.

\section{RESULTADOS E DISCUSSÃO}

Os resultados para as energias de interação estão resumidas nas Figuras 1 e 2. Além disso, há uma comparação com cálculos anteriores. ${ }^{26}$

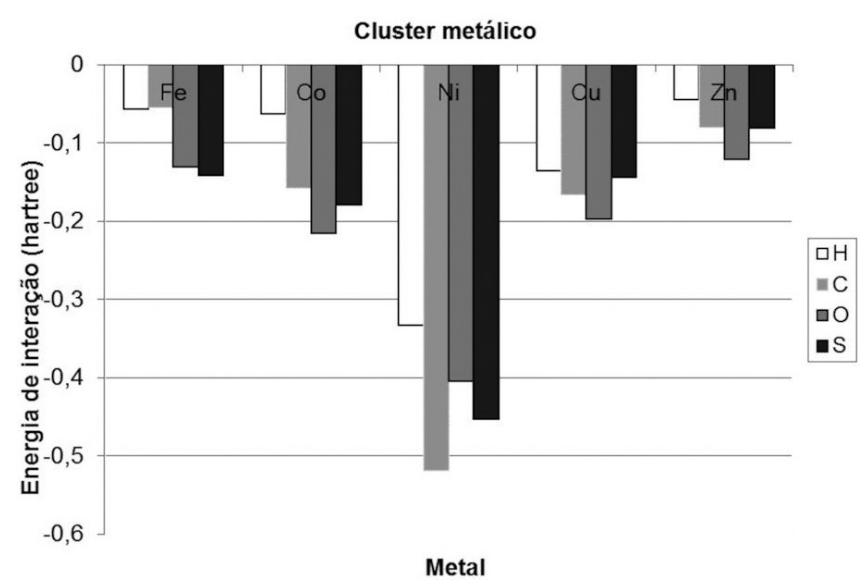

Figura 1. Energias de interação entre átomos pequenos e clusters metálicos da primeira série de transição usando o funcional híbrido B3LYP

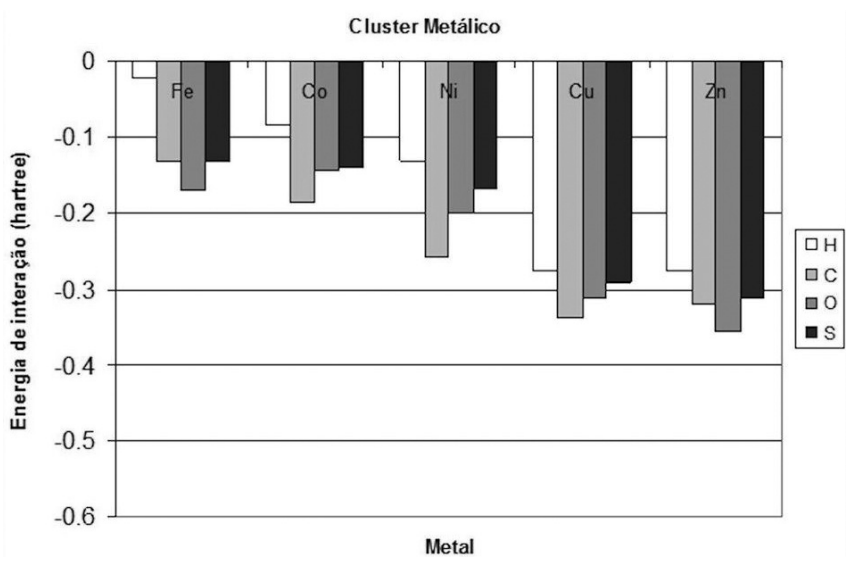

Figura 2. Energias de interação entre átomos pequenos e clusters metálicos da primeira série de transição usando o funcional híbrido XPBE96

As energias de adsorção foram determinadas a partir da Equação 8:

$$
E_{a d s}=E\left(M_{n} A\right)-\left[E\left(M_{n}\right)+E(A)\right]
$$

onde A é o átomo leve ( $\mathrm{H}, \mathrm{C}, \mathrm{O}$ ou $\mathrm{S})$ e $\mathrm{E}\left(\mathrm{M}_{\mathrm{n}} \mathrm{A}\right), \mathrm{E}\left(\mathrm{M}_{\mathrm{n}}\right)$ e $\mathrm{E}(\mathrm{A})$ representam a energia do cluster após a adsorção, a energia do cluster sem o átomo adsorvido e a energia do átomo livre, respectivamente.

\section{Observações relativas ao mecanismo de oxidação do hidrogênio sobre eletrodos metálicos}

Ao analisar-se os resultados das energias de adsorção para os átomos de $\mathrm{H}$ e $\mathrm{O}$ adsorvidos na superfície dos eletrodos metálicos, verifica-se que as mesmas tendências se manifestam em ambos os casos, numa correlação linear. Estes resultados indicam que os dois 
processos devem afetar de maneira significativa e semelhante as velocidades de reação para a oxidação do hidrogênio e, portanto, os resultados concordam totalmente com o mecanismo proposto por Ihara, ou seja, que as etapas que determinam a velocidade da reação ocorrem na superfície do metal. ${ }^{13}$ Apesar disso, outros mecanismos que consideram processos que ocorrem nas superfícies metálicas não podem ser descartadas (por exemplo, a formação de grupos $\mathrm{HO}_{\mathrm{Ni}}$ ) no estudo cinético. ${ }^{8,9,14}$ Além disso, os resultados obtidos não indicam mecanismos que consideram a transferência do átomo de hidrogênio da superfície do metal, como a etapa que determina a velocidade da reação (ou seja, o hydrogen spillover). Como o eletrólito é fundamental para este tipo de interação, como representa a Equação 3, este último mecanismo não pode ser completamente descartado, especialmente em eletrodos tipo cermet com alta afinidade por hidrogênio. ${ }^{11,12}$

Ao comparar-se os resultados da Figura 2 com a atividade obtida por Setoguchi et al., para anodos metálicos, infere-se que as energias de adsorção para $\mathrm{O}$ e $\mathrm{H}$ seriam altas, o que tornaria a etapa 3 (Equação 6) mais lenta no mecanismo proposto por Ihara (nesta etapa em particular, os átomos de $\mathrm{H}$ e $\mathrm{O}$ reagiriam, formando moléculas de $\mathrm{H}_{2} \mathrm{O}$ ), por outro lado, para o caso do $\mathrm{Ni}$, estas energias teriam valores intermediários, o que permitiria que a terceira etapa não fosse tão lenta. De acordo com o princípio de Sabatier, a atividade catalítica de uma superfície depende de um equilíbrio entre sua atividade e a resistência ao envenenamento em relação aos intermediários de reação, i.e., o catalisador ideal deve ser atraído pelos intermediários com força moderada. ${ }^{16,33}$ Este princípio é expresso geralmente na forma de uma curva do tipo vulcano e os resultados obtidos confirmam esta tendência. É importante ressaltar que para $\mathrm{Cu}$ e Zn, os módulos das energias de adsorção calculadas por B3LYP são consideravelmente menores, o que está de acordo com os dados empíricos que revelam que estes metais não são bons catalisadores para a oxidação do $\mathrm{H}_{2} \cdot{ }^{6,8,18}$

A fim de se estudar os problemas da deposição do carbono à adsorção do enxofre, pode-se considerar que as energias calculadas por B3LYP também possuem boa concordância com resultados previamente relatados, que mostram que o $\mathrm{Fe}$, $\mathrm{Co}$ e $\mathrm{Ni}$ apresentam uma tendência maior a sofrerem envenenamento por estes átomos, enquanto o $\mathrm{Cu}$ é menos suscetível. ${ }^{18,21,22}$ No caso específico do $\mathrm{Zn}$, não há relato e, pelos resultados obtidos, este metal seria ainda mais resistente ao envenenamento do que o $\mathrm{Cu}$, entretanto, a atividade catalítica do Zn é muito baixa, o que impede seu uso como anodo em uma célula a combustível.

No presente estudo, determinou-se a posição de equilíbrio de átomos pequenos $(\mathrm{H}, \mathrm{C}, \mathrm{O}$ e $\mathrm{S})$ adsorvidos sobre substratos metálicos. Em todos os cálculos, a posição inicial do átomo pequeno foi escolhida aleatoriamente. Observou-se que o comportamento dos átomos adsorvidos é diverso em relação aos substratos metálicos. Dois tipos de arranjo final foram encontrados: uma geometria piramidal na qual o átomo leve se localiza no topo de um sítio vazio formado por $\mathrm{n}$ átomos metálicos (semelhante ao sítio fcc em cristais) e, um arranjo externo, no qual o átomo adsorvido fica próximo a uma aresta do cluster metálico. As Figuras 3 e 4 apresentam exemplos destes resultados. No material suplementar são apresentadas todas as geometrias obtidas.

Verificou-se que os átomos leves apresentam uma tendência maior a ficarem na posição de vértice de uma pirâmide, sobre o sítio vazio (semelhante ao sítio fcc de cristais), mas o enxofre e o hidrogênio saem desta tendência com mais frequência, algumas vezes ocupando as posições próximas às arestas dos clusters. No final dos cálculos, observou-se que todos os átomos ficam fora dos clusters, o que está de acordo com o observado por Wang et al. usando uma abordagem semelhante..$^{25}$

Além disso, as distâncias entre os átomos leves e as superfícies sobre as quais eles interagem foram determinadas e estão listadas nas Tabelas 1 e 2 .

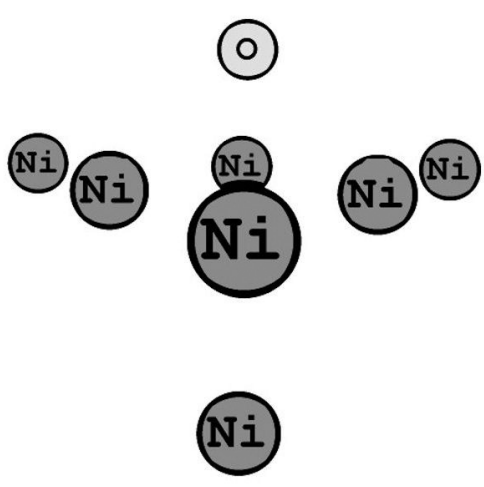

Figura 3. Geometria obtida para a adsorção de oxigênio sobre cluster de níquel usando-se o funcional híbrido B3LYP

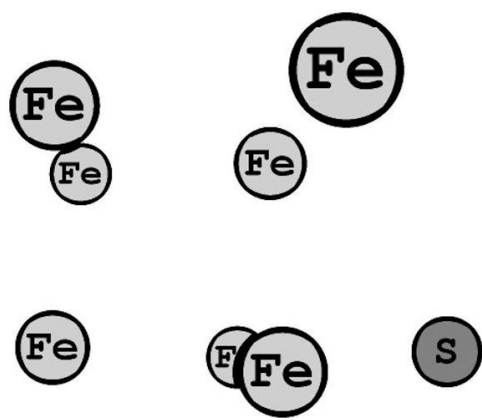

Figura 4. Geometria obtida para a adsorção de enxofre sobre cluster de ferro usando-se o funcional XPBE96

Tabela 1. Distâncias entre átomos adsorvidos e as das superfícies dos clusters metálicos em angstroms, calculadas usando-se o funcional B3LYP

\begin{tabular}{cccccc}
\hline & $\mathrm{Fe}$ & $\mathrm{Co}$ & $\mathrm{Ni}$ & $\mathrm{Cu}$ & $\mathrm{Zn}$ \\
\hline $\mathrm{H}$ & 1,116 & 1,152 & 1,166 & 0,976 & 1,309 \\
$\mathrm{C}$ & 1,060 & 1,082 & 1,027 & 1,364 & 1,515 \\
$\mathrm{O}$ & 1,187 & 1,318 & 1,287 & 1,356 & 1,308 \\
$\mathrm{~S}$ & 1,870 & 1,918 & 1,064 & 1,947 & 2,069 \\
\hline
\end{tabular}

Tabela 2. Distâncias entre átomos adsorvidos e as das superfícies dos clusters metálicos em angstroms, calculadas usando-se o funcional XPBE96

\begin{tabular}{lccccc}
\hline & $\mathrm{Fe}$ & $\mathrm{Co}$ & $\mathrm{Ni}$ & $\mathrm{Cu}$ & $\mathrm{Zn}$ \\
\hline $\mathrm{H}$ & 1,196 & 0,133 & 1,192 & 1,082 & 1,431 \\
$\mathrm{C}$ & 1,143 & 1,158 & 1,139 & 1,446 & 1,688 \\
$\mathrm{O}$ & 1,278 & 1,287 & 1,332 & 1,475 & 1,452 \\
$\mathrm{~S}$ & 1,939 & 1,935 & 1,963 & 2,105 & 2,210 \\
\hline
\end{tabular}

A última análise geométrica diz respeito às distâncias entre os átomos leves adsorvidos e os metais mais próximos e pode ser vista nas Tabelas 3 e 4.

Tabela 3. Distâncias entre átomos adsorvidos e os primeiros vizinhos metálicos, determinadas usando-se o funcional B3LYP, em angstroms

\begin{tabular}{lccccc}
\hline & $\mathrm{Fe}$ & $\mathrm{Co}$ & $\mathrm{Ni}$ & $\mathrm{Cu}$ & $\mathrm{Zn}$ \\
\hline $\mathrm{H}$ & 1,841 & 1,562 & 1,852 & 1,895 & 2,019 \\
$\mathrm{C}$ & 1,825 & 1,721 & 1,767 & 1,879 & 2,158 \\
$\mathrm{O}$ & 1,877 & 1,958 & 1,930 & 2,013 & 1,971 \\
$\mathrm{~S}$ & 2,395 & 2,404 & 2,301 & 2,442 & 2,477 \\
\hline
\end{tabular}


Tabela 4. Distâncias entre átomos adsorvidos e os primeiros vizinhos metálicos, determinadas usando-se o funcional XPBE96, em angstroms

\begin{tabular}{cccccc}
\hline & $\mathrm{Fe}$ & $\mathrm{Co}$ & $\mathrm{Ni}$ & $\mathrm{Cu}$ & $\mathrm{Zn}$ \\
\hline $\mathrm{H}$ & 1,848 & 1,665 & 1,868 & 1,827 & 2,088 \\
$\mathrm{C}$ & 1,878 & 1,793 & 1,828 & 2,010 & 2,284 \\
$\mathrm{O}$ & 1,916 & 1,865 & 1,961 & 2,083 & 2,049 \\
$\mathrm{~S}$ & 2,383 & 2,368 & 2,425 & 2,570 & 2,588 \\
\hline
\end{tabular}

A fim de se verificar se os resultados predizem a formação de ligações covalentes, comparamos nossos resultados com valores experimentais (Tabela 5) obtidos para hidretos, carbetos, óxidos e sulfatos. O uso de geometrias iniciais diferentes e o acompanhamento das curvas de energia potencial durante os cálculos de otimização geométrica permitem afirmar que as geometrias finais obtidas para os átomos leves são as de menor energia, ressalvando-se o fato de que os metais foram mantidos fixos em suas posições.

Tabela 5. Distâncias entre átomos pequenos e metais, determinadas experimentalmente, em angstroms ${ }^{34-49}$

\begin{tabular}{cccccc}
\hline & $\mathrm{Fe}$ & $\mathrm{Co}$ & $\mathrm{Ni}$ & $\mathrm{Cu}$ & $\mathrm{Zn}$ \\
\hline $\mathrm{H}$ & 1,896 & 1,793 & 1,870 & & \\
$\mathrm{C}$ & 1,899 & 1,911 & 1,861 & & \\
$\mathrm{O}$ & 1,944 & 2,216 & 1,922 & 1,940 & 2,106 \\
$\mathrm{~S}$ & 2,419 & 2,458 & 2,322 & 2,415 & 2,371 \\
\hline
\end{tabular}

A comparação entre os resultados observados e os dados empíricos mostra que os valores calculados concordam com as distâncias encontradas em sulfetos e óxidos. Ou seja, o método é capaz de prever as distâncias de interação metal-enxofre e metal-oxigênio. Há uma forte indicação de que a metodologia prevê a formação de ligações covalentes durante a adsorção. Uma vez mais, fica evidente a grande afinidade do $\mathrm{Fe}$, Co e Ni por oxigênio, o que reforça os resultados de energia de adsorção que indicam o mecanismo de oxygen spillover como o mais apropriado para explicar a atividade catalítica de anodos feitos com estes metais. Estes resultados também são consistentes com a maior tendência destes três metais de sofrerem envenenamento por enxofre, enquanto o cobre e o zinco são menos propensos a formarem ligações covalentes com o enxofre, o que diminui as chances de envenenamento, como observado para anodos de cobre. ${ }^{50}$

As discrepâncias obtidas nos cálculos realizados usando-se o funcional XPBE96 podem ser atribuídas ao seu caráter de funcional "puro", ou seja, aquele que não considera os efeitos de correlação e não superestima a ligação covalente..$^{51}$

\section{CONCLUSÃO}

A adsorção de átomos pequenos (H, C, O e S) sobre superfícies de metais da primeira série de transição foi investigada via DFT usando os funcionais B3LYP e XPBE96. As energias e as geometrias obtidas foram analisadas e comparadas a resultados previamente relatados. De acordo com os resultados obtidos, pode-se demonstrar que para clusters de sete átomos, a adsorção é favorecida para o carbono e o oxigênio. Para o hidrogênio, a interação é sempre fraca, especialmente no caso de $\mathrm{Cu}$ e $\mathrm{Zn}$.

Os átomos de oxigênio e carbono são adsorvidos em superfícies metálicas, enquanto o enxofre e o hidrogênio também interagem sobre as arestas dos clusters.

O estudo dos metais de transição entre ferro e cobre mostra uma relação linear entre a diferença de eletronegatividade entre metal e átomo adsorvido e as energias de interação, mas tal relação não existe no caso dos clusters de zinco.

No caso específico do cobre, é válida a comparação entre os resultados obtidos e os de Wang et al. usando o funcional B3LYP. A mesma ordem nas energias de adsorção foi encontrada $(\mathrm{C}>\mathrm{O}>\mathrm{S}>\mathrm{H}){ }^{25}$

Os resultados apresentados quando usados na discussão sobre anodos de células a combustível conduzem às seguintes conclusões: a atividade medida experimentalmente por Setoguchi et al. indica que o níquel deve possuir um desempenho melhor, o que é explicado pelo comportamento do tipo Vulcano. Este metal apresenta energias de adsorção intermediárias entre $\mathrm{H}$ e $\mathrm{O}$, como confirmam os valores obtidos por B3LYP. ${ }^{16}$ Este resultado é consistente como princípio de Sabatier, ou seja, o catalisador deve possuir uma atividade tal que permita afinidade pelos reagentes e a saída dos produtos.

$\mathrm{O}$ problema da deposição de carbono é mais grave para $\mathrm{Fe}, \mathrm{Co}$ e $\mathrm{Ni}$, de acordo com a literatura, e menos problemático para o $\mathrm{Cu}$, o que confirma os resultados obtidos para a adsorção de carbono, usando-se B3LYP. ${ }^{18}$

$\mathrm{O}$ envenenamento por enxofre também é crítico para Fe, Co e Ni. Os resultados confirmam a formação de sulfetos pelas distâncias metal-enxofre e energias de adsorção.

Os cálculos realizados validam a abordagem escolhida para estudar estes sistemas e podem ser usados na interpretação de dados experimentais no que concerne ao funcionamento de células a combustível, ao menos em termos qualitativos. A principal vantagem desta abordagem é a sua simplicidade quando comparada às usadas em outros trabalhos recentes. Dos funcionais empregados, o B3LYP apresentou um desempenho mais próximo aos resultados reportados na literatura.

\section{MATERIAL SUPLEMENTAR}

Disponível em http://quimicanova.sbq.org.br, na forma de arquivo PDF, com acesso livre.

\section{AGRADECIMENTOS}

Ao apoio financeiro do CNPq e da FAPERJ.

\section{REFERÊNCIAS}

1. Neurock, M.; J. Catal. 2003, 216, 73.

2. Parreira, R. L. T.; Caramori, G. F.; Galembeck, S. E.; Huguenin, F.; J. Phys. Chem. A 2008, 112, 11731.

3. Sholl, D. S.; Steckel, J.; Density Functional Theory: A Practical Introduction, $1^{\text {st }}$ ed., John Wiley \& Sons, Inc.: New Jersey, 2009.

4. Ingram, D. B.; Linic, S.; J. Electrochem. Soc. 2009, 156, 1457.

5. Ferrin, P.; Simonetti, D.; Kandoi S.; Kunkes, E.; Dumesic, J. A.; Nørskov, J. K.; Mavrikakis M.; J. Am. Chem. Soc. 2009, 131, 5809.

6. Sun, C.; Stimming, U.; J. Power Sources 2007, 171, 247.

7. Horita, T.; Kishimoto, H.; Yanaji, K.; Xiong, Y.; Sakai, N.; Brito, M. E.; Yokokawa, H.; Solid State Ionics 2006, 177, 1941.

8. Rossmeisl, J.; Bessler, W. G.; Solid State Ionics 2008, 178, 1694.

9. Bessler, W. G.; Solid State Ionics 2005, 176, 997.

10. Lu, X. C.; Zhu, J. H.; Bi, Z. H.; Solid State Ionics 2009, 180, 265.

11. Jiang, S. P.; Badawal, S. P. S.; Solid State Ionics 1999, 123, 209.

12. Jiang, S. P.; Badawal, S. P. S.; J. Eletrochem. Soc. 1997, 144, 3777.

13. Ihara, M.; Kusano, T.; Yokoyama, C.; J. Electrochem. Soc. 2001, 148, A209.

14. Bieberle, A.; Gauckler, L. J.; Solid State Ionics 2002, 146, 23.

15. Mizusaki, J.; Yamamura, T.; Mori, N.; Tagawa, H.; Proc. of the $17^{\text {th }}$ Ris $\phi$ Int. Symp. on Mat. Sci.: High Temperature Electrochemistry: Ceramics and Metals, Roskilde, Dinamarca, 1996. 
16. Setoguchi, T.; Okamoto, K.; Eguchi, K.; Arai, H.; J. Eletrochem. Soc. 1992, 139, 2875

17. Amado, R. S.; Malta, L. F. B.; Garrido, F. M. S.; Medeiros, M. E.; Quim. Nova 2007, 30, 189 .

18. Gross, M. D.; Vohs, J. M.; Gorte, R. J.; J. Mater. Chem. 2007, 17, 3071

19. Nikolla, E.; Schwank, J. W.; Linic, S.; Catal. Today 2008, 136, 243.

20. Offer, G. J.; Mermelstein, J.; Brightman, E.; Brandon, N. P.; J. Am. Ceram. Soc. 2009, 92, 763.

21. Choi, Y. M.; Compson, C.; Lin, M. C.; Liu, M.; J. Alloys Compd. 2007 427, 25.

22. Choi, Y. M. ; Compson, C.; Lin, M. C.; Liu, M.; Chem. Phys. Lett. 2006, $421,179$.

23. Cheng, Z.; Liu, M.; Solid State Ionics 2007, 178, 925.

24. Dong, J.; Cheng, Z.; Zha, S.; Liu, M.; J. Power Sources 2006, 156, 461

25. Wang, G.; Jiang, L; Cai, Z.; Pan, Y.; Guan, N.; Wu, Y.; Zhao, X.; Li, Y.; Sun, Y.; Zhong, B.; J. Mol. Struct. 2002, 589, 371.

26. Wang, L.; Electrochem. Commun. 2007, 9, 2212.

27. Pang, X.; Xue, L.; Wang, G.; Langmuir 2007, 23, 4910.

28. May, M.; Gonzalez, S.; Illas, F.; Surf. Sci. 2008, 602, 906.

29. Open Crystallography Database, http://www.crystallography.net, acessada em Julho 2009.

30. Becke, A. D.; J. Chem. Phys. 1993, 98, 5648.

31. Perdew, J. P.; Burke, K.; Ernzerhof, M.; Phys. Rev. Lett. 1996, 77, 3865.

32. Bylaska, E. J.; de Jong, W. A.; Govind, N.; Kowalski, K.; Straatsma, T. P.; Valiev, M.; Wang, D.; Apra, E.; Windus, T. L.; Hammond, J.; Nichols, P.; Hirata, S.; Hackler, M. T.; Zhao, Y.; Fan, P.-D.; Harrison, R. J.; Dupuis, M.; Smith, D. M. A.; Nieplocha, J.; Tipparaju, V.; Krishnan, M.; Wu, Q.; van Voorhis, T.; Auer, A. A.; Nooijen, M.; Brown, E.; Cisneros, G.; Fann, G. I.; Fruchtl, H.; Garza, J.; Hirao, K.; Kendall, R.; Nichols, J. A.; Tsemekhman, K.; Wolinski, K.; Anchell, J.; Bernholdt, D.; Borowski, P.; Clark, T.; Clerc, D.; Dachsel, H.; Deegan, M.; Dyall, K.; Elwood, D.; Glendening, E.; Gutowski, M.; Hess, A.; Jaffe, J.; Johnson, B.; Ju, J.; Kobayashi, R.; Kutteh, R.; Lin, Z.; Littlefield, R.; Long, X.; Meng, B.; Nakajima, T.; Niu, S.; Pollack, L.; Rosing, M.; Sandrone, G.; Stave, M.; Taylor, H.; Thomas, G.; van Lenthe, J.; Wong, A.; Zhang, Z.;
NWChem, A Computational Chemistry Package for Parallel Computers, Version 5.1, Estados Unidos, 2007.

33. Bligaard, T.; Norshov, J. K.; Dahl, S.; Matthiesen, J.; Christensen, C. H.; Sehested, S.; J. Catal. 2004, 224, 206.

34. Antonov, V. E.; Baier, M.; Dorner, B.; Fedotov, V. K.; Grosse, G.; Kolesnikov, A. I.; Ponyatovsky, E. G.; Schneider, G.; Wagner, F. E.; J. Phys.: Condens. Matter 2002, 14, 6427.

35. Wood, I. G.; Vočadio, L.; Knight, K. S.; Dobson, D. P.; Marshall, W. G.; Price, D.; Brodholt, J.; J. Appl. Crystallogr. 2004, 37, 82.

36. Maslen, E. N.; Streltsov, V. A.; Streltsova, N. R.; Acta Crystallogr., Sect. $B$ 1994, 50, 435.

37. Fasiska, E. J.; Phys. Status Solidi A 1972, 10, 169.

38. Fedotov, V. K.; Antonov, V. E.; Antonova, T. E.; Bokhenkov, E. L.; Dorner, B.; Gosse, G.; Wagner, F. E.; J. Alloys Compd. 1999, 291, 1.

39. Clarke, J.; Chem. Ind. (London, U.K.) 1951, 46, 1004.

40. Taylor, D.; Trans. J. Br. Ceram. Soc. 1984, 83, 5.

41. Barthelemy, E.; Carcaly, C.; J. Solid State Chem. 1987, 66, 191.

42. Somenkov, V. A.; Journal of the Less-Common Metals 1987, 129, 171.

43. Nakagura, C.; J. Phys. Soc. Jpn. 1958, 13, 1005.

44. Tarascon, J. M.; Vaughan, G.; Chabre, Y.; Seguin, L.; Anne, M.; Strobel, P.; Amatucci, G.; J. Solid State Chem. 1999, 147, 410.

45. Kolkmeijer, N. H.; Moesveld, A. L.; Z. Kristallogr. 1931, 80, 91.

46. Ehrenberg, H.; McAllister, J. A.; Marshall, W. G.; Attfield, J. P.; J. Phys.: Condens. Matter. 1999, 11, 6501.

47. Gasymov, G. B.; Asadov, Y. G.; Guseinov, G. G.; Belov, N. V.; Dokl. Akad. Nauk. SSSR. 1978, 239, 846.

48. Cui, S.; Feng, W.; Hu, H.; Feng, Z.; Wang, Y.; J. Alloys Compd. 2009, 476, 306.

49. Yim, W. M.; J. Appl. Phys. 1969, 40, 2617.

50. He, H.; Gorte, R. J.; Vohs, J. M.; Electrochem. Solid-State Lett. 2005, 8, 279.

51. Jones, R. O. Em Computational Nanoscience: Do It Yourself!; Grotendorst, J.; Blügel, S.; Marx, D., eds.; John von Neumann Institute for Computing, Jülich 2006, vol. 31, p. 45. 


\section{INTERAÇÃO DE ÁTOMOS LEVES COM CLUSTERS DE METAIS DE TRANSIÇÃO}

\section{Eduardo Pires Cassús*}

CENPES - Petrobras, Av. Horácio de Macedo, 950, 21941-915 Rio de Janeiro - RJ, Brasil

Sérgio de Paula Machado, Francisco M.S. Garrido, Marta E. Medeiros e Juan Omar Machuca-Herrera

Departamento de Química Inorgânica, Instituto de Química, Universidade Federal do Rio de Janeiro, CP 68563, 21945-970 Rio de Janeiro - RJ, Brasil

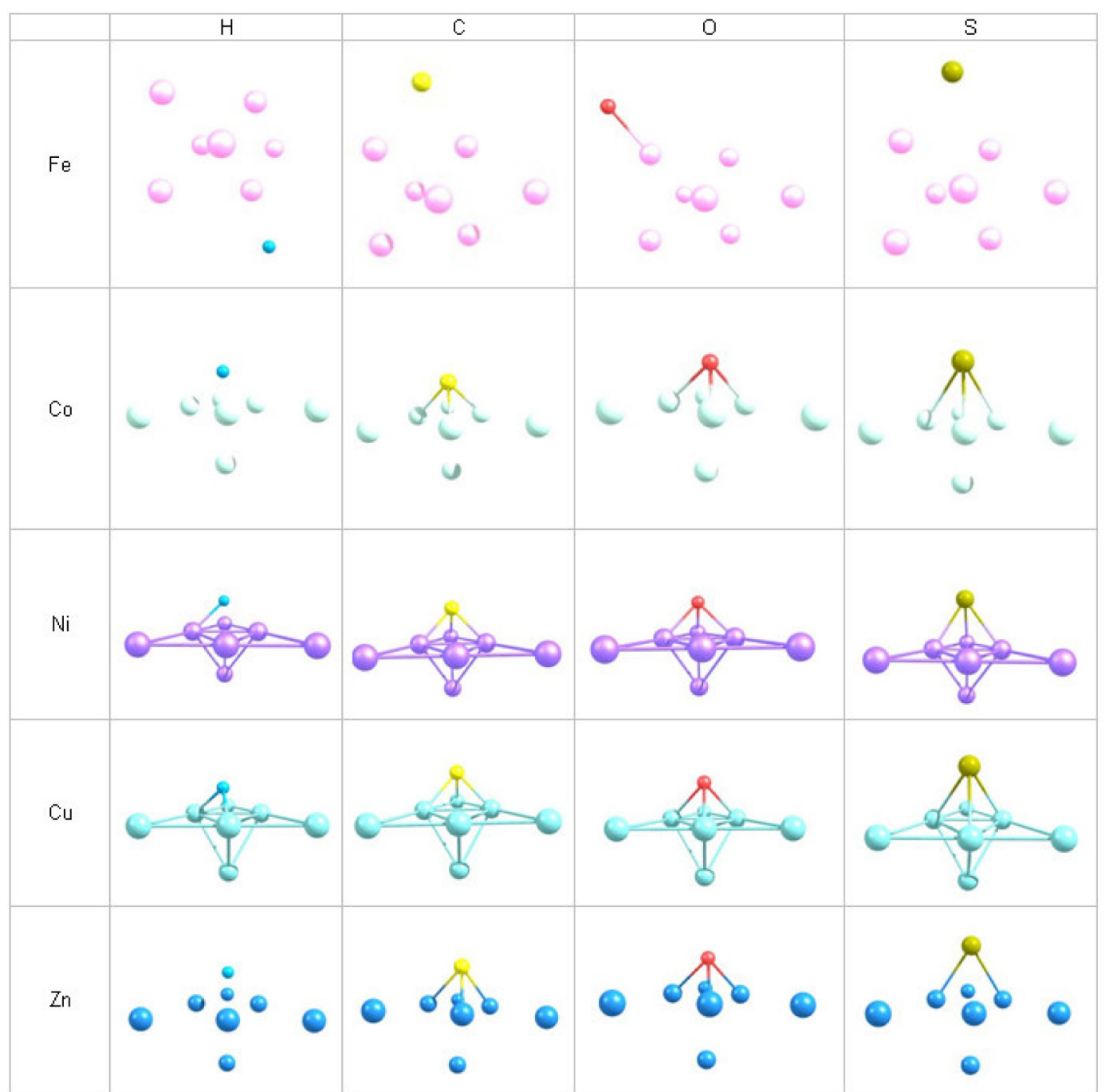

Figura 1S. Geometrias obtidas para a adsorção de átomos de H, C, O e S sobre clusters de ferro, cobalto, níquel, cobre e zinco usando-se o funcional híbrido $B 3 L Y P$ 


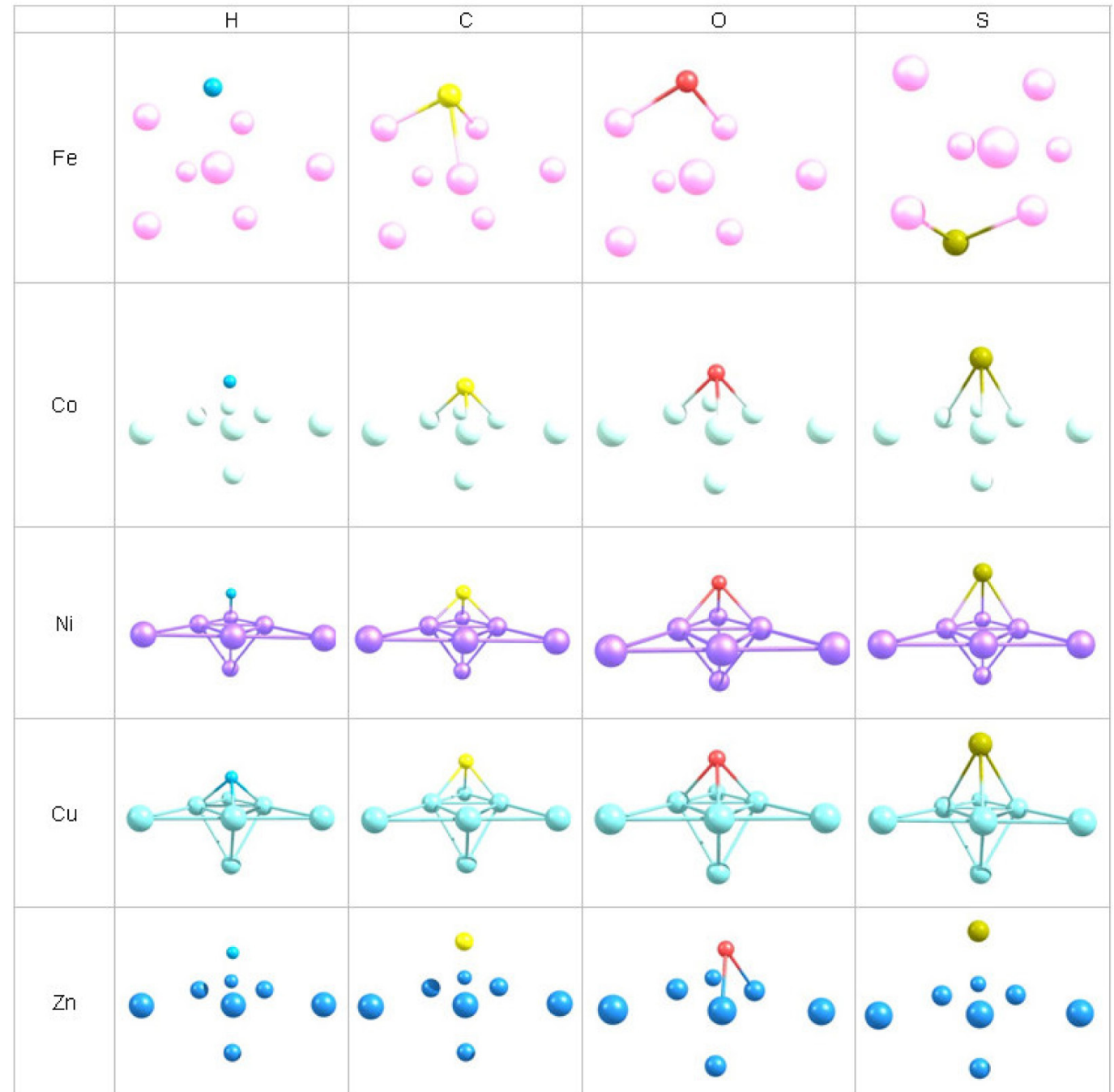

Figura 2S. Geometrias obtidas para a adsorção de átomos de H, C, O e S sobre clusters de ferro, cobalto, níquel, cobre e zinco usando-se o funcional XPBE96 